\title{
Spin-orbit coupling effect by minority interface resonance states in single-crystal magnetic tunnel junctions
}

\author{
Y. Lu, ${ }^{1,{ }^{*}}$ H.-X. Yang, ${ }^{2}$ C. Tiusan, ${ }^{1,3}$ M. Hehn, ${ }^{1}$ M. Chshiev, ${ }^{2}$ A. Duluard,${ }^{1}$ B. Kierren, ${ }^{1}$ G. Lengaigne, ${ }^{1}$ D. Lacour, ${ }^{1}$ \\ C. Bellouard, ${ }^{1}$ and F. Montaigne ${ }^{1}$ \\ ${ }^{1}$ Institut Jean Lamour, UMR 7198, CNRS-Nancy Université, BP 239, 54506 Vandoeuvre, France \\ ${ }^{2}$ SPINTEC, UMR 8191 CEA/CNRS/UJF-Grenoble 1/G-INP, 38054 Grenoble, France \\ ${ }^{3}$ Centre for Superconductivity, Spintronics and Surface Science (C4S), Technical University of Cluj-Napoca, \\ Str. Memorandumului nr. 28, 400114 Cluj-Napoca, Romania
}

(Received 11 January 2012; published 16 November 2012)

\begin{abstract}
Spin-orbit coupling (SOC) related to the minority interface resonance states (IRSs) of $\mathrm{Fe}(001)$ has been evidenced in full-epitaxial $\mathrm{Fe}(001) / \mathrm{MgO} / \mathrm{Fe}$ magnetic tunnel junctions (MTJs) from magnetotransport experiments correlated to theoretical ab initio calculations. We show that the SOC effect strongly depends on the symmetry composition of the IRS, which has been skillfully engineered via the interfacial chemical structure in the $\mathrm{Fe}(001) / \mathrm{MgO}$ stack. The SOC scattering is enhanced between the majority $\Delta_{1}$ and minority $\Delta_{5}$ band near the Fermi level when presenting a $\Delta_{5}$ symmetry dominant IRS at the $\mathrm{Fe} / \mathrm{MgO}$ interface. Our results validate the interplay between SOC and IRSs, which gives further understanding of the mechanisms responsible for the tunneling anisotropic magnetoresistance and the large perpendicular magnetic anisotropy at the $\mathrm{Fe} / \mathrm{MgO}$ interface.
\end{abstract}

Interfacial engineering in single-crystal magnetic tunnel junctions (MTJs) has been of extensive interest since it allows experimental and theoretical investigation and understanding of fundamental physics related to the spin-dependent tunneling. Among these systems, the single-crystal $\mathrm{Fe}(001) / \mathrm{MgO} / \mathrm{Fe}$ MTJ represents a model system where the Bloch-state-symmetry-dependent spin filtering leads to a huge tunnel magnetoresistance (TMR) effect. ${ }^{1,2}$ In addition, the interface resonant states (IRSs) at the $\mathrm{Fe} / \mathrm{MgO}$ interface also have an important influence on the spin transport properties. ${ }^{3-7}$ Recently, the large perpendicular magnetic anisotropy (PMA) values have been reported experimentally in $\mathrm{CoFeB} / \mathrm{MgO}$ structures, ${ }^{8,9}$ which is of extreme interest to the next-generation high-density PMA memory. These results have also been supported theoretically, ${ }^{10,11}$ with reports that a very large PMA up to $3 \mathrm{erg} / \mathrm{cm}^{2}$ can be obtained in $\mathrm{Fe} / \mathrm{MgO} / \mathrm{Fe}$ MTJ by calculating the spin-orbit coupling (SOC)-induced energy splitting around the Fermi level $\left(E_{F}\right),{ }^{10}$ which is even larger than the $\mathrm{Co} / \mathrm{Pt}$ or $\mathrm{Co} / \mathrm{Pd}$ interface. ${ }^{12}$ As known, the IRSs may produce, via the SOC mechanism, tunneling anisotropic magnetoresistive (TAMR) effects in MTJs based on $\mathrm{Fe}(001)$ electrodes. ${ }^{13-16}$ Therefore, it seems that a common mechanism responsible for the TAMR and PMA effects is the SOC at the $\mathrm{Fe}(001) / \mathrm{MgO}$ interface and related to the minority surface state of $\mathrm{Fe}(001)$.

In this work, we have studied the interplay of the SOC and minority IRSs on the magnetotransport properties in singlecrystal $\mathrm{Fe}(001) / \mathrm{MgO} / \mathrm{Fe}$ MTJs with planar magnetization configuration, and their impact on the TAMR and the PMA at $\mathrm{Fe} / \mathrm{MgO}$ interface. We show that the SOC effect is strongly dependent on the Bloch symmetry of the IRS. To obtain IRSs with different symmetry character, ${ }^{17}$ we used skillful chemical interface engineering to fabricate MTJs with two types of bottom $\mathrm{Fe} / \mathrm{MgO}$ interfaces: without and with fully ordered half-monolayer carbon doping. By correlated experimental and theoretical analysis, we have found that the SOC is strongly enhanced between the majority $\Delta_{1}$ and minority $\Delta_{5}$ band near $E_{F}$ when the IRS at the $\mathrm{Fe} / \mathrm{MgO}$ interface presents a $\Delta_{5}$ dominating symmetry.

The MTJs were elaborated within a molecular beam epitaxy (MBE) system with the following structure: $\mathrm{MgO}(100)$ substrate//alternative $\mathrm{MgO}(3 \mathrm{~nm})$ seed layer $/ \mathrm{Fe}(45 \mathrm{~nm}) /$ $\mathrm{MgO}(2.5 \mathrm{~nm}) / \mathrm{Fe}(10 \mathrm{~nm}) / \mathrm{Co}(20 \mathrm{~nm}) / \mathrm{Au}(10) .{ }^{18}$ The "alternative" 3-nm $\mathrm{MgO}$ seed layer acts as an antidiffusion barrier to block the carbon diffusion from the substrate to the bottom $\mathrm{Fe} / \mathrm{MgO}$ interface during the annealing. On the sample unprotected by the $\mathrm{MgO}$ seed layer, the segregation of $\mathrm{C}$ induces a $\mathrm{C}(2 \times 2)$ reconstruction on the bottom Fe surface after annealing at $450{ }^{\circ} \mathrm{C}$, as certified by reflecting high-energy electron diffraction (RHEED) patterns along the $\mathrm{Fe}[100]$ direction (see Ref. 18). Finally, micrometric-size MTJs were fabricated by UV lithography. The magnetotransport measurements have been performed by a dc two-probe configuration, where the negative bias corresponds to the electrons tunneling from the top to bottom electrode.

At a low bias of $10 \mathrm{mV}$, we have measured high TMR ratios above $290 \%$ for both samples at $10 \mathrm{~K}$, which is attributed to the symmetry-dependent filtering approach in the high-quality $\mathrm{Fe} / \mathrm{MgO} / \mathrm{Fe}$ MTJs. ${ }^{1,2}$ At room temperature (RT), the sample without $\mathrm{C}$ shows a higher TMR (160\%) compared to the sample with C (122\%). To identify the IRS which is located in the spin minority channel, we have performed the inelastic electron tunneling spectroscopy (IETS) experiments through the derivative of the dynamic conductance in the antiparallel (AP) state. As is well established, one can easily identify the minority IRS at the bottom $\mathrm{Fe} / \mathrm{MgO}$ interface ${ }^{5}$ in the AP state at negative bias when the electrons tunnel from the majority occupied states at the top interface to the minority unoccupied states at the bottom interface. Figures 1(a) and 1(b) show the $d^{2} I / d V^{2}$ curves in an AP configuration for both samples at different temperatures. For the sample without $\mathrm{C}$ [Fig. 1(a)], one can observe several peaks located at $\pm 0.03,-0.16$, and $-1.0 \mathrm{~V}$, respectively. The peaks at $\pm 0.03 \mathrm{~V}$ are considered to be related to the interfacial magnon excitation ${ }^{19,20}$ [magnified 

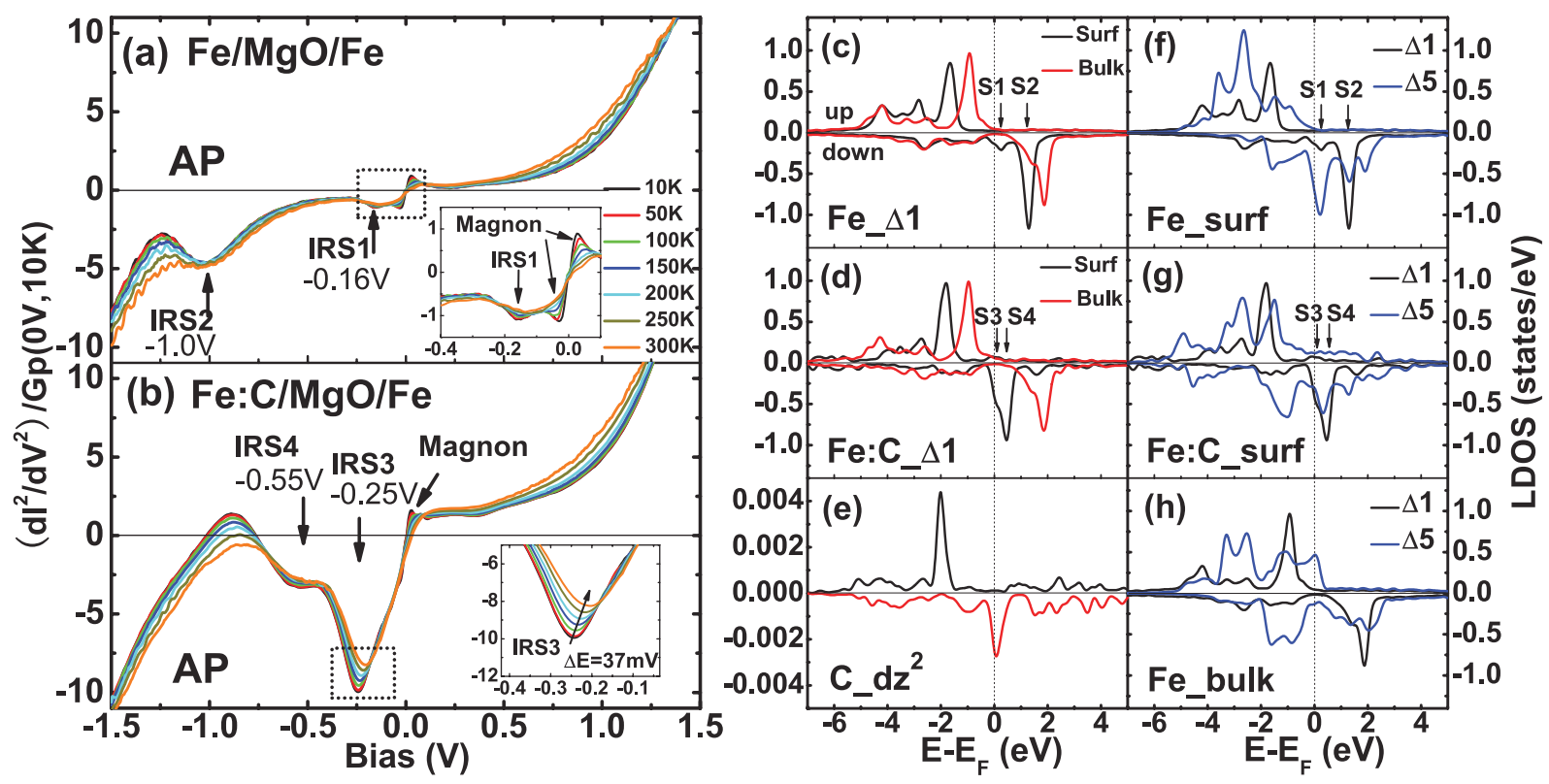

FIG. 1. (Color online) $d^{2} I / d V^{2}$ curves measured at different temperatures in an AP configuration for MTJs (a) without C and (b) with C. The insets show the magnified zoom in the dashed square. LDOS in $\Delta_{1}$ symmetry for bcc Fe(001) slabs (c) without C and (d) with C. (e) LDOS in $d_{z 2}$ symmetry for surface C. Comparison of LDOS in $\Delta_{1}$ and $\Delta_{5}$ symmetry for surface Fe layer (f) without C, (g) with C, and (h) bulk Fe.

inset of Fig. 1(a)]. This magnon excitation with spin-flip events increases the AP conductance and results in a reduction of TMR. The peaks at $-0.16 \mathrm{~V}$ (IRS1) and $-1.0 \mathrm{~V}(\mathrm{IRS} 2)$ are attributed to be the IRSs at the bottom $\mathrm{Fe} / \mathrm{MgO}$ interface. These two IRS peaks were also recently evidenced by Zermatten et al. in single-crystal $\mathrm{Fe} / \mathrm{MgO} / \mathrm{Fe}$ MTJs. ${ }^{5}$ However, there are no IRS peaks that appear at positive bias, and this confirms that the local density of states (LDOS) at the top interface mainly exhibits bulk characters because of the relatively rough top $\mathrm{MgO} / \mathrm{Fe}$ interface which quenches the $\mathrm{Fe}(001) \mathrm{IRS}^{4,21}$ With the increase of $T$, the IRS peaks gradually disappear, while remaining at the same position in voltage. This means that the temperature has a strong influence on the IRS at the clean $\mathrm{Fe} / \mathrm{MgO}$ interface. For the sample with $\mathrm{C}$ [Fig. 1(b)], two strong IRS peaks at $-0.25 \mathrm{~V}$ (IRS3) and $-0.55 \mathrm{~V}$ (IRS4) were found. Indeed, as further confirmed by our $a b$ initio analysis, the IRS intensity is strongly enhanced with respect to the case of the pure $\mathrm{Fe} / \mathrm{MgO}$ interface due to the interfacial hybridization of $\mathrm{Fe}$ and $\mathrm{C}$. With the increase of $T$, the two IRS peaks exhibit different behaviors. IRS3 shifts by $37 \mathrm{mV}$ towards zero bias from $10 \mathrm{~K}$ to $300 \mathrm{~K}$, while IRS4 has no shift with $T$. This shift could be related to the thermal-induced distance change between the $\mathrm{C}$ and $\mathrm{Fe}$ atoms, as already evidenced from angle-resolved photoemission experiments on the noble metal (111) surface where the temperature-induced variation of the bulk lattice constant can shift the surface-state energy in the bulk band gaps. ${ }^{22}$

To improve our understanding and to study the symmetry composition of IRSs, we have performed spin-polarized firstprinciples calculations on an Fe surface with and without $\mathrm{C}$ by using the Vienna ab initio simulation package (VASP). ${ }^{23}$ To simplify the structure, we have constructed a bcc Fe supercell (slab) of volume $2.866 \times 2.866 \times 57.215 \AA$ with 16 layers of bcc Fe and a large vacuum space. Doped carbon atoms are located on the first layer between Fe atoms with a structure of $(1 \times 1)$. We focus first on the LDOS in $\Delta_{1}$ symmetry since the $\Delta_{1}$ Bloch states have the smallest decay rate into the tunnel barrier and have the highest contribution to the tunnel current. ${ }^{1}$ Figures 1(c) and 1(d) show, respectively, that the surface and bulk LDOS belong to $\Delta_{1}$ symmetry for the two types of samples. For the pure Fe(001) slab [Fig. 1(c)], one can find that the bulk minority peak at $1.85 \mathrm{eV}$ is shifted to $1.30 \mathrm{eV}$ (S2) in the surface Fe layer, and an additional surface minority peak appears at $0.25 \mathrm{eV}(\mathrm{S} 1)$. These two peaks agree well with the two IRS peaks in Fig. 1(a). When doped with carbon [Fig. 1(d)], a strong minority peak at $0.48 \mathrm{eV}$ (S4) appears and a shoulder peak at $0.13 \mathrm{eV}(\mathrm{S} 3)$ is enhanced close to $E_{F}$, which are also in good agreement with the two IRS peaks found in Fig. 1(b). In Fig. 1(e), the LDOS for the surface carbon atom shows a sharp $d_{z 2}$ peak right above (almost crossing) $E_{F}$, which explains that the enhanced surface state peak is resulted from the hybridization of $\mathrm{Fe}$ and $\mathrm{C}$ atom orbits. We then present, in Figs. 1(f)-1(h), the comparison of LDOS projected in $\Delta_{1}$ and $\Delta_{5}$ symmetries for Fe bulk and for a surface without and with $\mathrm{C}$, respectively. For the surface free of $\mathrm{C}$, a strong minority $\Delta_{5}$ peak appears close to $E_{F}$, corresponding to the peak position of $\mathrm{S} 1$ in $\Delta_{1}$ symmetry. This peak is also identified by the calculations with the quasiparticle self-consistent GW method. ${ }^{24}$ This validates that the IRS1 has a significant $\Delta_{5}$ composition. On the contrary, at the position of S2, the LDOS of $\Delta_{5}$ symmetry character is strongly reduced, indicating that IRS2 has a dominant $\Delta_{1}$ composition. This is in good agreement with Ref. 5, where the authors conclude towards a $\Delta_{1}$ dominant character for IRS2 from the analysis of the IRS attenuation rate in MTJs with different $\mathrm{MgO}$ barrier thickness. It is worth mentioning that recently Bonell et al. ${ }^{25}$ have also identified one two-dimensional IRS peak at $1 \mathrm{eV}$ above $E_{F}$. By angle- and spin-resolved photoemission measurement, this 


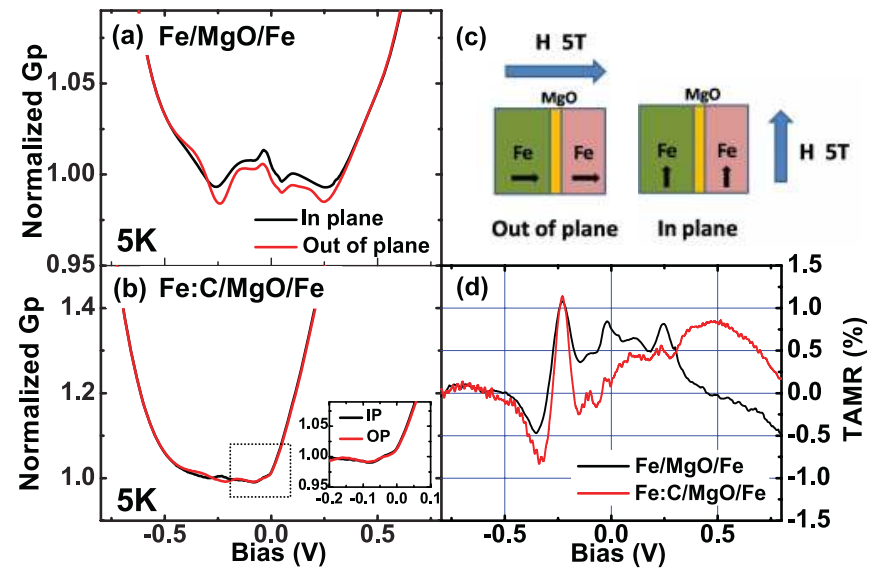

FIG. 2. (Color online) Bias dependence of normalized dynamic conductance in a perpendicular and in-plane field at $5 \mathrm{~K}$ for (a) a pure Fe MTJ and (b) a C-doped MTJ. The inset shows the magnified zoom in the dashed square. (c) Schematic diagram of the TAMR measurement. (d) Bias dependence of TAMR for MTJs with and without C.

peak shows a $\Delta_{1}$ dominant feature, which agrees well with our experimental and calculation results for IRS2. For the surface doped with C, only a small minority $\Delta_{5}$ LDOS peak is found in the vicinity of $E_{F}(\mathrm{~S} 3)$, which also indicates that the IRSs in the C-doped sample have a $\Delta_{1}$ dominant character. Compared with Fe surface states, the bulk Fe LDOS show very small $\Delta_{5}$ composition in the minority channel around $E_{F}$. However, a strong $\Delta_{5}$ composition is found in the majority channel near the $E_{F}$ and quickly reduces when the energy is higher than $0.2 \mathrm{eV}$ above $E_{F}$, which can be understood by the top of the $\Delta_{5}$ band in the $\mathrm{Fe}(001)$ majority band structure [see the inset of Fig. 3(c)].

To demonstrate the $\mathrm{SOC}$ at the $\mathrm{Fe} / \mathrm{MgO}$ interface, we have carried out TAMR measurements on the two types of samples in a physical property measurement system (PPMS). As schematically shown in Fig. 2(c), a $5 T$ magnetic field is applied at $5 \mathrm{~K}$ from the in-plane and out-of-plane direction to check the difference of the dynamic conductance. In both cases, the $5 T$ field is sufficiently large to saturate the magnetization of $\mathrm{Fe}$ electrodes parallel to the field. Figure 2(a), for the pure Fe MTJ, clearly shows a large difference of conductance between the two field directions within the range of $\pm 0.2 \mathrm{~V}$. The in-plane conductance is higher than the out-of-plane one. However, in our C-doped sample [Fig. 2(b)], the small difference in conductivity between the two directions indicated a weak SOC around the zero bias [magnified in the inset of Fig. 2(b)]. Figure 2(d) shows the bias dependence of TAMR, which is defined as $\left(G_{I P}-G_{O P}\right) / G_{O P}$. Although the C-doped sample shows a similar peak at $-0.25 \mathrm{eV}$ as the pure Fe MTJ, the TAMR is much weaker at zero bias. In addition, the $\approx 1 \%$ of TAMR in our single-crystalline $\mathrm{Fe} / \mathrm{MgO} / \mathrm{Fe}$ system is $2-3$ times higher than that of polycrystalline $\mathrm{CoFe} / \mathrm{MgO} / \mathrm{CoFe}$ MTJs, and 10 times higher than that of MTJs with an amorphous $\mathrm{Al}_{2} \mathrm{O}_{3}$ barrier, ${ }^{13}$ which validates the theoretic predication that the IRS can enhance the TAMR due to the shift of the resonant surface band via the Rashba effect when the magnetization direction changes and this Rashba effect is produced by the SOC at the interface. ${ }^{14,15}$ The different TAMR at zero bias clearly indicates that the sample without $\mathrm{C}$ has stronger SOC at the interface than the sample with C.

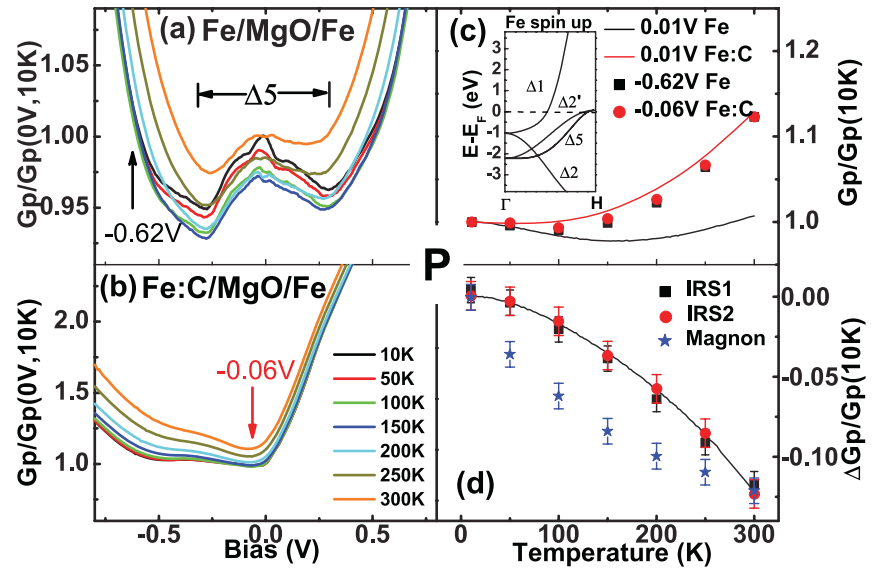

FIG. 3. (Color online) Bias-dependent $G_{P}$ at low bias at different $T$ for MTJs (a) without $\mathrm{C}$ and (b) with C. (c) Variation of normalized $G_{P}$ vs $T$ at bias of $10 \mathrm{mV}$ (solid lines), $-0.62 \mathrm{~V}$ without C (black squares), and $-0.06 \mathrm{~V}$ with $\mathrm{C}$ (red circles). Inset: band structure of majority spin in bcc bulk $\mathrm{Fe}(001)$ along the $\Gamma H$ direction. (d) The difference of normalized $G_{P}$ vs $T$ for the samples with and without C. The variation of peak intensities of IRS1, IRS2, and magnon [marked in Fig. 1(a)] are scaled to match the $\Delta G_{P}$ temperature-variation curve.

To further clarify the different TAMR behaviors in the two types of samples, we measured the bias dependence of the parallel dynamic conductance $\left(G_{P}\right)$ at different temperatures with in-plane magnetization. In Fig. 3(a), the MTJ without C presents a conductivity bump between $\pm 0.2 \mathrm{eV}$, in which range we have observed large TAMR in Fig. 2(a). This conductivity bump with local minima at $\pm 0.2 \mathrm{eV}$ is generally suggested to reflect the dispersion of the majority energy bands of bulk $\mathrm{Fe}(001) .{ }^{18}$ As shown in the inset of Fig. 3(c), the top of the $\Delta_{5}$ band lies at $0.2 \mathrm{eV}$ above $E_{F}$. When the energy of the hot electrons arriving across the barrier overcomes the top of the $\Delta_{5}$ band, the conduction channel associated with $\Delta_{5}$ symmetry quenches. Therefore, the bump in $G_{P}$ validates the contribution of the majority $\Delta_{5}$ electrons to the tunneling at low bias, superimposed on the paraboliclike $\Delta_{1}$ conductance background. However, for the sample with $\mathrm{C}$ [Fig. 3(b)], we cannot find any local minima around $\pm 0.2 \mathrm{eV}$. This would indicate a reduced contribution of the $\Delta_{5}$ channel compared with the one of the $\Delta_{1}$ channel. To further confirm this point, we have plotted in Fig. 3(c) the temperature variation of the normalized $G_{P}$ for the sample with $\mathrm{C}$ at $-0.06 \mathrm{~V}$ [red arrow in Fig. 3(b)] and for the sample without $\mathrm{C}$ at $-0.62 \mathrm{~V}$ [black arrow in Fig. 3(a)], where the pure $\Delta_{1}$ conductance is expected from the parabolic curve shape. Interestingly, their temperature variation can be matched perfectly, which proves that the bump-related $\Delta_{5}$ conductance is quenched in the sample with $\mathrm{C}$. To further explore the temperature dependence of the conductivity bump, we plot the normalized $G_{P}$ vs $T$ at a fixed bias of $10 \mathrm{mV}$ in Fig. 3(c). For the sample with C, the $G_{P}$ show a monotonous increase of $12 \%$ from 10 to $300 \mathrm{~K}$. This can be understood from the thermal excitation of the electrons around $E_{F}$ in the electrode. ${ }^{26}$ However, the conductance for the sample without $\mathrm{C}$ first decreases to a minimum at about $150 \mathrm{~K}$, and then increases with $T$. If we carefully check this nonmonotonous behavior in Fig. 3(a), we can find that the decrease of $G_{P}$ before $150 \mathrm{~K}$ is correlated to the decrease of 
(a)

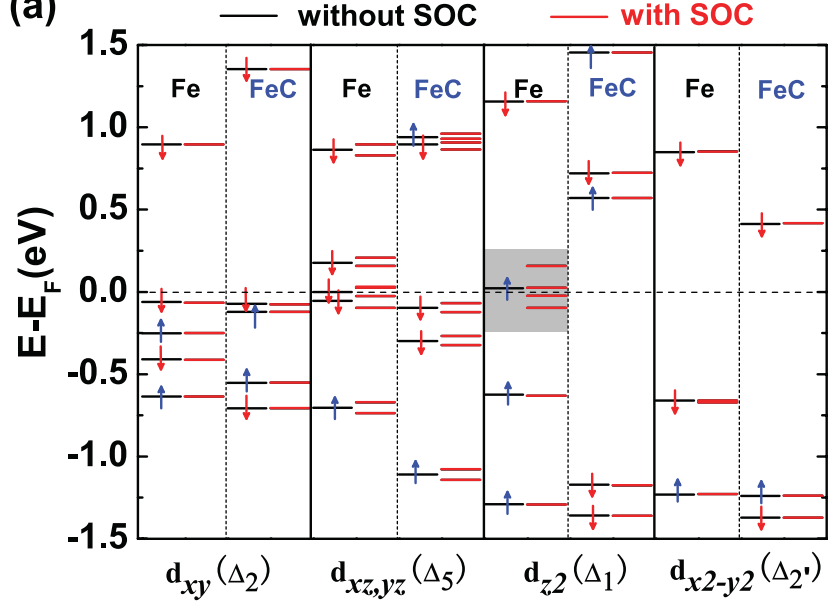

(b)

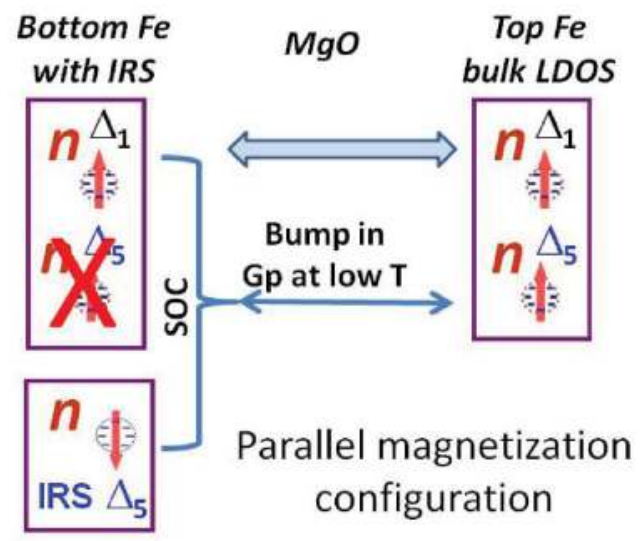

FIG. 4. (Color online) (a) SOC effect on wave-function character at the $\bar{\Gamma}$ point of the interfacial $\mathrm{Fe} d$ orbitals for the pure Fe/MgO and $\mathrm{Fe}: \mathrm{C} / \mathrm{MgO}$ interface. The left subcolumns show the energy levels without SOC (with marks of spin-up and spin-down), and the right subcolumns show the energy levels after taking account of SOC (out-of-plane magnetization). The gray zone indicates the new energy levels generated with SOC. (b) Schematic illustration of the SOC between the majority $\Delta_{1}$ and minority $\Delta_{5}$ band, which results in the bump in $G_{P}$ at low temperature.

the bump in $G_{P}$. Then with the increase of the background $\Delta_{1}$ conductance, the total $G_{P}$ increases even if the bump is continuously decreasing with $T$. If we consider that there is no (neglecting) bump contribution in the $G_{P}$ of the sample with $\mathrm{C}$, we can roughly extract the bump contribution in the sample without $\mathrm{C}$ by taking the difference of $G_{P}$ between the two samples $\left(\Delta G_{P}\right)$. As shown in Fig. 3(d), this extracted bump contribution $\left(\Delta G_{P}\right)$ monotonously decreases with increasing $T$, which can be well matched with the intensity change of IRS1 and IRS2, which is marked in Fig. 1(a), but not with the magnon peak. This gives important evidence that the conductance-bump variation in $T$ would be influenced by the IRS rather than by the magnons. However, the following questions still remain: (i) How can the minority IRS influence the conductivity bump reflecting the majority $\Delta_{5}$ conductance, and (ii) why does the bump-related $\Delta_{5}$ conductance signature in $G_{P}$ disappear for the sample with C?

To answer these questions and understand the role of IRSs on the bump in $G_{P}$, we have performed ab initio calculations by taking account of SOC for pure and C-doped $\mathrm{Fe} / \mathrm{MgO}$ interfaces. Here we used periodic junction structures with five layers of $\mathrm{Fe}$ and three layers of $\mathrm{MgO}$. Here, $\mathrm{C}$ is doped at the interface similar to our previous structure for additional oxygen at the $\mathrm{Fe} / \mathrm{MgO}$ interface. ${ }^{27}$ More details can be found in Ref. 10. Figure 4(a) shows the band structure around $E_{F}$ at the $\bar{\Gamma}$ point of the interfacial $\mathrm{Fe} d$ orbitals for the pure $\mathrm{Fe} / \mathrm{MgO}$ and $\mathrm{Fe}: \mathrm{C} / \mathrm{MgO}$ interface without and with SOC (out-of-plane magnetization). Let us concentrate on the band levels of $d_{x z, y z}$ and $d_{z 2}$ for pure $\mathrm{Fe} / \mathrm{MgO}$ interface in the immediate vicinity of $E_{F}$. When no SOC is included (left subcolumns), there are six double degenerated band levels with $d_{x z}$ and $d_{y z}$ characters, which represent the minority Bloch states with $\Delta_{5}\left(p_{x}, p_{y}, d_{x z}\right.$, $\left.d_{y z}\right)$ symmetry. At the same time, there is a band level resulting from the hybridization between $\mathrm{Fe}-d_{z 2}$ and $\mathrm{O}-p_{z}$ orbitals that possesses a signature of the majority $\Delta_{1}\left(s, p_{z}, d_{z 2}\right)$ symmetry, which is the heart of the spin-filtering phenomenon causing enhanced TMR values in MgO-based MTJs. ${ }^{1}$ When SOC is switched on (right subcolumns), one can clearly see that the degeneracy is lifted for energy levels with a $d_{x z, y z}$ orbital character. Meanwhile, these levels become hybridized with a Fe- $d_{z 2}$ orbital, resulting in the appearance of additional levels of both $d_{z 2}$ and $d_{x z, y z}$ orbital character. We have marked in the gray zone the new energy levels generated with $\Delta_{1}$ symmetry after taking account of SOC. The entire mechanism can be seen as spin-orbit-induced mixing between the majority $\Delta_{1}$ and the minority $\Delta_{5}$ Bloch states. Apparently, the SOC effect is very weak in the case of a Fe: $\mathrm{C} / \mathrm{MgO}$ interface since there are no energy levels generated in the $\Delta_{1}$ channel because of the $\Delta_{1}$ dominant character of IRSs. The PMA value can then be calculated from the energy difference after taking account of SOC for outof-plane and in-plane magnetization. A large PMA value up to $2.93 \mathrm{erg} / \mathrm{cm}^{2}$ confirms the strong SOC for the pure $\mathrm{Fe} / \mathrm{MgO}$ interface. ${ }^{10}$ On the contrary, a very small PMA of about 0.015 $\mathrm{erg} / \mathrm{cm}^{2}$ is obtained at the $\mathrm{Fe}: \mathrm{C} / \mathrm{MgO}$ interface. This result shows that the out-of-plane components of $d_{x z, y z}$ orbitals of IRSs play a crucial role for the PMA at the $\mathrm{Fe} / \mathrm{MgO}$ interface.

The strong SOC at the pure $\mathrm{Fe} / \mathrm{MgO}$ interface allows the minority $\Delta_{5}$ IRS states to mix with the majority $\Delta_{1}$ states and generate new states in the majority $\Delta_{1}$ and $\Delta_{5}$ channels (mixture of $\Delta_{1}$ and $\Delta_{5}$ symmetry and also mixture of spin-up and spin-down). These new states will easily couple with the abundant majority $\Delta_{5}$ states, as shown in Fig. 1(h) at $E_{F}$ of the top $\mathrm{MgO} / \mathrm{Fe}$ interface where a bulk $\mathrm{Fe}(001)$ property is considered. This explains the experimentally observed conductivity bump relating to the majority $\Delta_{5}$ conductance in $G_{P}$ at low temperature. We schematically show this mechanism in Fig. 4(b) with neglecting the contributions from the bulk minority and $\Delta_{2}, \Delta_{2^{\prime}}$ channels. With the increase of $T$, the intensity of the IRS decreases due to the phonon excitation, so that this SOC effect also decreases, leading to the decrease of $\Delta_{5}$ conductance. We can extend our explanation and argue that the quenching of the bump-related $\Delta_{5}$ conductance in the sample with $\mathrm{C}$ can be attributed to the weaker SOC effect due to the $\Delta_{1}$ dominant symmetry of minority IRSs. Finally, 
the large TAMR at zero bias in the sample without $\mathrm{C}$ can also be understood with the same mechanism. The enhancement of in-plane conductance is attributed to this SOC-related $\Delta_{1}$ to $\Delta_{5}$ band scattering due to the $\Delta_{5}$ symmetry of IRSs, which opens an additional conductivity channel. In the out-of-plane configuration, the Rashba effect lifts the spin degeneracy of the surface states ${ }^{14}$ and consequently reduces the SOC-related additional conductivity.

In summary, we have found that the SOC is strongly enhanced when the IRS at the $\mathrm{Fe} / \mathrm{MgO}$ interface presents a $\Delta_{5}$ symmetry dominant character. Our work gives deep experimental and theoretical insight into the interplay between the SOC and the minority-spin IRS at the interface, which is responsible for the TAMR effects in the single-crystal MTJ with $\mathrm{Fe}(001)$ electrodes and the large PMA at the $\mathrm{Fe}(001) / \mathrm{MgO}$ interface.

We thank T. Hauet and S. Suire for their help with the TAMR measurement. C.T. acknowledges the SPINCHAT project (ANR-07-BLAN-341) and POS CCE ID. 574 (SMIS-CSNR 12467). H.X.Y. and M.C. acknowledge support by the Grenoble Nanosciences Foundation Chair of Excellence Program.
*Corresponding author: yuan.lu@ijl.nancy-universite.fr

${ }^{1}$ W. H. Butler, X.-G. Zhang, T. C. Schulthess, and J. M. MacLaren, Phys. Rev. B 63, 054416 (2001); J. Mathon and A. Umerski, ibid. 63, 220403(R) (2001).

${ }^{2}$ S. Yuasa et al., Nature Mater. 3, 868 (2004).

${ }^{3}$ K. D. Belashchenko, J. Velev, and E. Y. Tsymbal, Phys. Rev. B 72, 140404(R) (2005).

${ }^{4}$ C. Tiusan, J. Faure-Vincent, C. Bellouard, M. Hehn, E. Jouguelet, and A. Schuhl, Phys. Rev. Lett. 93, 106602 (2004).

${ }^{5}$ P.-J. Zermatten, G. Gaudin, G. Maris, M. Miron, A. Schuhl, C. Tiusan, F. Greullet, and M. Hehn, Phys. Rev. B 78, 033301 (2008).

${ }^{6}$ C. Heiliger, P. Zahn, B. Y. Yavorsky, and I. Mertig, Phys. Rev. B 72, 180406(R) (2005).

${ }^{7}$ I. Rungger, O. Mryasov, and S. Sanvito, Phys. Rev. B 79, 094414 (2009).

${ }^{8}$ S. Ikeda et al., Nature Mater. 9, 721 (2010).

${ }^{9}$ W. X. Wang et al., Appl. Phys. Lett. 99, 012502 (2011).

${ }^{10}$ H. X. Yang, M. Chshiev, B. Dieny, J. H. Lee, A. Manchon, and K. H. Shin, Phys. Rev. B 84, 054401 (2011).

${ }^{11}$ M. K. Niranjian et al., Appl. Phys. Lett. 96, 222504 (2010).

${ }^{12}$ K. Yakushiji et al., Appl. Phys. Lett. 97, 232508 (2010).

${ }^{13}$ L. Gao, X. Jiang, S.-H. Yang, J. D. Burton, E. Y. Tsymbal, and S. S. P. Parkin, Phys. Rev. Lett. 99, 226602 (2007).
${ }^{14}$ A. N. Chantis, K. D. Belashchenko, E. Y. Tsymbal, and M. van Schilfgaarde, Phys. Rev. Lett. 98, 046601 (2007).

${ }^{15}$ M. N. Khan et al., J. Phys.: Condens. Matter 20, 155208 (2008).

${ }^{16}$ J. Moser, A. Matos-Abiague, D. Schuh, W. Wegscheider, J. Fabian, and D. Weiss, Phys. Rev. Lett. 99, 056601 (2007).

${ }^{17}$ C. Uiberacker and P. M. Levy, Phys. Rev. B 64, 193404 (2001).

${ }^{18}$ C. Tiusan, M. Sicot, M. Hehn, C. Belouard, S. Andrieu, F. Montaigne, and A. Schuhl, Appl. Phys. Lett. 88, 062512 (2006); C. Tiusan, M. Sicot, J. Faure-Vincent, M. Hehn, C. Bellouard, F. Montaigne, S. Andrieu, and A. Schuhl, J. Phys.: Condens. Matter 18, 941 (2006); C. Tiusan, F. Greullet, M. Hehn, F. Montaigne, S. Andrieu, and A. Schuhl, ibid. 19, 165201 (2007).

${ }^{19}$ Y. Ando et al., Appl. Phys. Lett. 87, 142502 (2005).

${ }^{20}$ V. Drewello, J. Schmalhorst, A. Thomas, and G. Reiss, Phys. Rev. B 77, 014440 (2008).

${ }^{21}$ V. Serin et al., Phys. Rev. B 79, 144413 (2009)

${ }^{22}$ R. Paniago et al., Surf. Sci. 336, 113 (1995).

${ }^{23}$ G. Kresse and J. Hafner, Phys. Rev. B 47, 558 (1993); G. Kresse and J. Furthmüller, ibid. 54, 11169 (1996); J. P. Perdew, K. Burke, and M. Ernzerhof, Phys. Rev. Lett. 77, 3865 (1996).

${ }^{24}$ S. V. Faleev et al., arXiv:1010.4086v1.

${ }^{25}$ F. Bonell et al., Phys. Rev. Lett. 108, 176602 (2012).

${ }^{26}$ R. Stratton, J. Phys. Chem. Solids 23, 1177 (1962).

${ }^{27}$ H. X. Yang et al., Appl. Phys. Lett. 96, 262509 (2010). 